dependent-magnetic resonance imaging (BOLD-MRI) was used to quantify changes in renal oxygenation. Tissue expression and distribution of RLN receptor (RXFP1) was determined by qPCR and immunofluorescence. Expression of vasoconstrictor genes was quantified by qPCR array.

Results RXFP1 was detected on glomerular podocytes, renal pericytes, and endothelial cells of the renal, segmental and interlobar

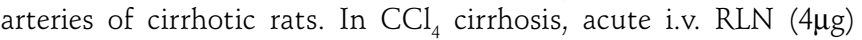
induced a $50 \%$ increase in RBF after 60 minutes $(p<0.01$ vs. placebo, $n=6$ ). BOLD-MRI showed increased tissue oxygenation at the same timepoint in renal cortex and medulla. Extended s.c. RLN increased $\mathrm{RBF}$ by $54 \%$ in $\mathrm{CCl}_{4}(p<0.01$ vs. placebo, $n=8)$ and $57 \%$ in BDL ( $p<0.001$ vs. placebo, $n=5)$ and increased GFR by $138 \%$ in $\mathrm{CCl}_{4}(p<0.01$ vs. placebo, $n=8)$ and $103 \%$ in BDL $(p<0.05$ vs. placebo, $n=5$ ). Mean arterial pressure was unaffected by RLN. L-NAME $(250 \mathrm{mg} / \mathrm{L})$ orally (p.o.) abrogated the effect of RLN on RBF and GFR. The relative expression of vasoconstrictor genes in the kidney was markedly reduced by RLN treatment.

Conclusion RLN increases RBF in experimental cirrhosis. Crucially, RLN also improves renal function and oxygenation but does not induce systemic hypotension even in decompensated disease. The effects of RLN are mediated via augmentation of $\mathrm{NO}$ and downregulation of vasoconstrictor genes known to be important in the pathogenesis of HRS. RLN has potential as a treatment for HRS and further translational studies are warranted.

Disclosure of Interest None Declared.

\section{PWE-147 HUMAN HERPESVIRUS AND ADENOVIRUS UNIQUE GENETIC SEQUENCES DETECTED IN HEPATOCELLULAR CANCER GENOMES}

doi:10.1136/gutjnl-2013-304907.435

1."W Fateen, ${ }^{2} \mathrm{~S}$ Berri, ${ }^{2} \mathrm{H}$ Wood, ${ }^{3} \mathrm{~J}$ Morgan, ${ }^{3} \mathrm{G}$ Taylor, ${ }^{1} \mathrm{P}$ Quirke. ${ }^{1}$ Pathology and Tumour Biology: '2Section of Pre-Cancer Genomics, '3 Section of Translational Genomics, University of Leeds, Leeds, UK

Introduction Hepatitis $\mathrm{C}$ virus ( $\mathrm{HCV}$ ) is the most common cause of hepatocellular cancer (HCC) in the western world. HCV is an RNA virus that does not integrate with human DNA and so the oncogenic mechanisms of HCV remain unclear. Next generation sequencing (NGS) provides a flexible platform and generates large amounts of data at a relatively small time and constantly reducing costs. The role of viral infection is well established in the aetiology of a wide range of tumours. In this study we investigate DNA of HCV driven HCC for the possibility of integration of all known viral genomes.

Methods Bar-coded DNA libraries from 41 samples of various stages of development of HCC from 6 different patients were sequenced in parallel using NGS. One to two million $74 \mathrm{bp}$ reads per genome were generated. The reads were aligned to all known viral genomes downloaded from the National Center for Biotechnology Information using Burrows-Wheeler Aligner (BWA). Reads with mapping scores of $<37$ were discarded. Basic Local Alignment Search Tool (BLAST) was used to test if the sequences that aligned to viral genomes belonged to the human genome or any other viruses apart from the identified virus. Only those reads were the BWA alignment matched the leading BLAST hit were considered

Results Six test samples mapped to unique sequences from Human herpesvirus 6 . The test samples included a single HCC and 5 premalignant nodules from 2 different patients. Six test samples mapped to unique sequences of Human Adenovirus (6/41). The test samples in this case included 4 HCCS and a 2 premalignant nodules from 2 different patients. A single dysplastic nodule mapped to Human papillomavirus.

Conclusion DNA from HCV driven HCC was searched for all viral genome sequences only Human Herpes 6, Human Adenovirus and Human pappilomavirus were found in a small number of cases.
Further studies are needed to understand their relation to HCV hepatocarcinogenesis.

Disclosure of Interest None Declared.

\section{PWE-148 HEPATOTOXICITY FROM ANABOLIC ANDROGENIC STEROIDS MARKETED AS DIETARY SUPPLEMENTS: CONTRIBUTION FROM ATP8B1/ABCB11 MUTATIONS?}

doi:10.1136/gutjnl-2013-304907.436

1." ${ }^{7}$ Elsherrif, ${ }^{1,2} \mathrm{~J}$ R Potts, ${ }^{3} \mathrm{M}$ R Howard, ${ }^{1} \mathrm{~A}$ Barnardo, ${ }^{1} \mathrm{~S}$ Cairns, ${ }^{4} \mathrm{~A}$ S Knisely, ${ }^{1,2} \mathrm{~S}$ Verma. ${ }^{1}$ Department of Gastroenterology and Hepatology, Brighton and Sussex University Hospitals; '2Department of Medicine, Brighton and Sussex Medical School; ${ }^{3}$ Department of Pathology, Brighton and Sussex University Hospitals, Brighton; ${ }^{4}$ nstitute of Liver Studies, King's College Hospitals, London, UK

Introduction In the United Kingdom (UK) it is illegal to produce, supply, or possess androgenic anabolic steroids (AAS) with intent. Despite this, non-prescription use of AAS, often marketed as dietary supplements, persists. We encountered drug-induced liver injury (DILI) associated with use of AAS and attempted to elucidate underlying mechanisms.

Methods We describe two patients with cholestatic DILI following ingestion of the dietary supplement massdrol ("Celtic Dragon") containing the AAS 2a-17a-dimethyl-etiocholan-3-one,17b-ol.

Results Two Western European males (aged 25 and 45 years) presented to our institute between July, 2011, and March, 2012, with jaundice and intractable pruritus following use of massdrol acquired from fellow gym users. Screening found no other causes of hepatobiliary disease. Despite significant hyperbilirubinaemia (respective peaks: 614 and $229 \mu \mathrm{mol} / \mathrm{L})$, peak gamma glutamyl transferase activities were within "normal range". Besides "bland" intralobular cholestasis, consistent with DILI, liver biopsy in both found deficiency of canalicular expression of the ectoenzymes neutral endopeptidase (CD10), alanyl aminopeptidase (CD13), GGT, and carcinoembryonic antigen (CD66). This suggested generalised abnormality in ectoenzyme trafficking to, or retention within, canalicular membranes, as seen in ATP8B1 disease (familial intrahepatic cholestasis 1 [FIC1]). The younger patient showed normal expression of bile salt export pump (BSEP, encoded by $A B C B 11)$ and of multidrug resistance protein 3 (MDR3; encoded by $A B C B 4$ ); in the older BSEP but not MDR3 marking was focally diminished. While this may have been due to AAS-induced inhibition of expression of normal $A T P 8 B 1 / A B C B 11$, it also raised the intriguing possibility of mutation in either of these genes - in effect, that AAS exposure had triggered initial episodes of benign recurrent intrahepatic cholestasis type $1 / 2$. On sequencing, $A T P 8 B 1$ was normal in both patients; the younger was heterozygous for the mutation c.2093G > A mutation in $A B C B 11$, a known polymorphism previously encountered in association with intrahepatic cholestasis following antibiotic exposure (personal communication, R Thompson). Morbidity from cholestasis and pruritus was substantial, necessitating use of multiple antipruritic agents and consideration for extracorporal albumin dialysis (MARS). At last follow-up, however, jaundice was resolved in both.

Conclusion AAS marketed as dietary supplements remain a cause of serious DILI in the UK; underlying mechanisms remain speculative but may include unmasking of genetic cholestatic syndromes.

Disclosure of Interest None Declared.

\section{PWE-149 THE EFFICACY AND SAFETY OF TREATING HEPATITIS C IN PATIENTS WITH A DIAGNOSIS OF SCHIZOPHRENIA}

doi:10.1136/gutjnl-2013-304907.437

1, Z Mustafa, ${ }^{2} \mathrm{~J}$ Schofield, ${ }^{3} \mathrm{P}$ R Mills, ${ }^{3} \mathrm{M}$ Priest, ${ }^{3} \mathrm{R}$ Fox, ${ }^{4} \mathrm{~S}$ Dutta, ${ }^{5} \mathrm{~J}$ Morris, ${ }^{1} \mathrm{E}$ Forrest, ${ }^{1} \mathrm{R}$ Gillespie, ${ }^{1} \mathrm{~A}$ J Stanley, ${ }^{1 S}$ Barclay. 'Glasgow Royal Infirmary; ${ }^{2} \mathrm{NHS}$ Greater Glasgow g Clyde; ${ }^{3}$ Gartnavel General Hospital; " Victoria Infirmary; ${ }^{5}$ Southern General Hospital, Glasgow, UK 


\begin{tabular}{|c|c|c|c|}
\hline & Schizophrenia (n=22) & $\begin{array}{c}\text { Patients without } \\
\text { schizophrenia } \\
(\mathbf{n}=1453)\end{array}$ & $\mathrm{p}$ value \\
\hline $\begin{array}{c}\text { Discontinuation due to } \\
\text { side effects }\end{array}$ & $4.5 \%(1)$ & $13 \%(189)$ & $\mathrm{p}-0.34$ \\
\hline $\begin{array}{c}\text { Discontinuation due to } \\
\text { non-compliance }\end{array}$ & $0 \%(0)$ & $4.9 \%(72)$ & $\mathrm{p}=0.62$ \\
\hline $\begin{array}{c}\text { Overall Sustained } \\
\text { Viral Response (SVR) }\end{array}$ & $81.8 \%(18)$ & $54.2 \%(788)$ & $\mathrm{p}=0.09$ \\
\hline SVR in Genotype 1 & $33.3 \%(2 / 6)$ & $32.8 \%(199 / 555)$ & $\mathrm{p}=0.25$ \\
\hline SVR in Genotype 2\&3 & $100 \%(16 / 16)$ & $82 \%(589 / 714)$ & $\mathrm{p}-0.01$ \\
\hline
\end{tabular}

\section{Abstract PWE-149 Figure}

Introduction Treating hepatitis $\mathrm{C}$ with pegylated interferon alpha may induce or exacerbate psychiatric illness including depression, mania and aggressive behaviour. There is limited data regarding treatment in the context of chronic schizophrenia. We sought to establish the safety and efficacy of treatment of patients with a diagnosis of schizophrenia amongst patients attending treatment centres in Greater Glasgow

Methods Patient and treatment data collected on the Scottish hepatitis $C$ database were retrospectively analysed according to the presence or absence of a diagnosis of schizophrenia. Combination antiviral therapy was defined as Interferon (pegylated or standard) and Ribavirin. Treatment outcomes including sustained viral response (SVR) rates, reasons for treatment termination and adverse events were documented

Results 5497 patients were recorded on the database, of whom 64 $(1.2 \%)$ had a diagnosis of schizophrenia. Patients with and without schizophrenia were of similar age at diagnosis [median 34 (IOR $31-40$ ) vs 36 (IOR 29-41) years, $p=0.85$ ]. Patients with schizophrenia had higher rates of current or previous intravenous drug use [50/64 (78.1\%) vs 3015/5433 (55.5\%), p < 0.01] and prior alcohol excess > 21 units/week [25/64 (39\%) vs 1211/5433 (22.2\%), $\mathrm{p}=0.02)]$. More patients with schizophrenia had a diagnosis of cirrhosis [13/64 (20.3\%) vs 589/5419 (10.86\%), p = 0.02]. Of those patients who had attended at least one clinic appointment 1639/4415 (37.1\%) of patients without schizophrenia commenced treatment versus $26 / 61(42.6 \%)$ of patients with schizophrenia $(p=0.21)$. Patients with schizophrenia took almost three times as long to commence treatment after initial referral [median 1123 (IOR 531-2130) vs 421 (IOR 209-1086) days, $\mathrm{p}<0.01$ ], despite similar times from referral to first attendance [median 65 (IOR 36-141) vs 62 (IOR 35-130) days, $p=0.92]$ The treatment outcomes were as follows:

Conclusion Patients with stable schizophrenia are good candidates for hepatitis $C$ treatment

Disclosure of Interest None Declared.

\section{Neoplasia and cancer pathogenesis}

\section{PWE-150 “THE EARLY GROWTH RESPONSE GENES INDUCE APOPTOSIS IN COLON CANCER CELLS"}

doi:10.1136/gutjnl-2013-304907.438

1,"A Koulouris. ${ }^{1}$ Gastroenterology-Experimental Immunology, Queen Mary, University of London, London, UK

Introduction The Egr familly (Early Growth Respone Genes) of zinc finger transcription factors, which consists of four members; Egr-1, $-2,-3$ and -4 , have been proved to have dynamic functions in the regulation of cell growth and the immune responses.Moreover, in a number of malignancies-which is a cell growth and immune related process- it seems that Egr1 and 2 induce apoptosis leading to the inhibition of the tumour growth. The present study was designed to answer the question whether colon cancer cells undergo apoptosis when the EGR genes are exogenously introduced and if the presence of a mutant p53 can affect this apoptotic pathway.

Methods Two cell lines deriving from human colon cancer; one p53 negative (DLD1) and another p53 positive (HCT116) were transfected with Egr-1, -2 and -3 and a fluorescent protein which was the marker of the transfection. The transfected cells were incubated for 48 hours. Flow cytometry was used to create a pure population of transfected cells and 24hours later these cells were examined in the fluoroscopic microscope and compared with the controls.

Results We found that all three Egr members can suppress tumour cell growth suggesting that the function of Egr in the control of cell growth is not associated with the function of p53. In addition to the growth arrest, the transfected cells changed morphology to round shape indicating of senescence.

Conclusion This may suggest that Egr molecules are important to control the unwanted growth in response to malignant transformation. Our results not only demonstrated an important function of Egr molecules and also indicate the therapeutic potential for the treatment of tumour.

Disclosure of Interest None Declared.

\section{PWE-151 CELL-MEDIATED IMMUNE RECOGNITION OF CEA IS ASSOCIATED WITH EARLY TUMOUR RECURRENCE FOLLOWING RESECTION OF COLORECTAL CANCER}

doi:10.1136/gutjnl-2013-304907.439

${ }^{1} \mathrm{C}$ Brown, ${ }^{1} \mathrm{M}$ Scurr, ${ }^{2} \mathrm{G}$ Betts, ${ }^{1} \mathrm{~A}$ Gallimore, ${ }^{1,}$ 'A Godkin. ${ }^{1}$ Institute of Infection and Immunity, Cardiff University, Cardiff; ' ${ }^{N}$ uffield Department of Surgical Sciences, Oxford University, Oxford, UK

Introduction Colorectal cancer (CRC) is one of the commonest malignancies in men and women. Clinical staging is used to predict prognosis after resection. The interaction of the cancer with the adaptive cellular immune response plays an important role in disease pathogenesis, but this relationship may be compromised by a population of regulatory Foxp3 ${ }^{+} \mathrm{CD}^{+} \mathrm{T}$ cells (Tregs). Here, the 5 year post-operative clinical outcome was correlated with pre-operatively measured anti-tumour immune responses.

Methods Eighty patients with non-metastatic CRC, undergoing a resection with curative intent, were recruited over 24 months. CD4 ${ }^{+}$ $\mathrm{T}$ cell responses to tumour associated antigens (CEA and 5T4) were compared to control antigens (PPD and HA). The influence of immune regulation was measured by repeating the assays after in vitro depletion of Tregs. Clinical databases were interrogated for details, including morbidity and mortality data, and the five year overall survival (OS), time to progression (TTP), and progression free survival (PFS) was calculated. These parameters were compared to the original details of pre-operative anti-tumour immune responses. 